\title{
Fibrosis of mesothelial cell-induced peritoneal implantation of ovarian cancer cells
}

This article was published in the following Dove Press journal: Cancer Management and Research

\author{
Jinou Wang' \\ Chang Liu' \\ Xiaoying Chang' \\ Yafei Qi' \\ Zhi Zhu ${ }^{2}$ \\ Xianghong Yang'
}

'Department of Pathology, Shengjing Hospital of China Medical University, Shenyang, China; ${ }^{2}$ Department of Surgical Oncology, The First Hospital of China Medical University, Shenyang, China

Correspondence: Xianghong Yang Department of Pathology, Shengjing Hospital of China Medical University, Sanhao Street 36, Shenyang I I000I,

China

Tel +86 I894025 I640

Fax +86 249661575213

Email Yangxh@sj-hospital.org
Background: Peritoneal metastasis frequently occurs in patients with advanced ovarian cancer and is the main basis for a poor prognosis. The mechanism underlying preferential ovarian cancer spread to the peritoneum is not well understood.

Methods: Herein, we investigated the significance and mechanism underlying fibrosis of mesothelial cells promoting peritoneal implantation of ovarian cancer. We have assessed the mesothelial cell fibroblast transformation process in peritoneal tissues of omentum and fibrotic mesothelial cell release of chemokines to promote dissemination by scanning electron microscopy, ELISA, Western blot, and Transwell chamber assay.

Results: We showed that the fibrosis of mesothelial cells exists in the peritoneum of ovarian cancer patients with peritoneal metastasis. Fibrosis of the mesothelial cells was induced by TGF$\beta 1$, which upregulates the CXCL12-CXCR4 and CXCL16-CXCR6 axes of mesothelial cells. Conclusion: CXCL12-CXCR4 and CXCL16-CXCR6 may be important signaling pathways closely involved in peritoneal metastasis of ovarian cancer that require further investigation. The findings may lead to developing alternative strategies aimed at preventing and treating the metastasis of ovarian cancer.

Keywords: ovarian cancer, peritoneal, mesothelial cell, fibrosis

\section{Introduction}

Ovarian cancer is the most lethal gynecological malignancy. The majority of ovarian cancer patients are diagnosed when tumors have widely spread within the peritoneal cavity, which ultimately leads to mortality. ${ }^{1}$ Although peritoneal metastasis plays a crucial role in promoting tumor progression and decreasing patient survival rates, the mechanism underlying ovarian cancer spread has not been thoroughly explored. The "seed and soil" hypothesis is one of the important theories explaining peritoneal metastasis of ovarian cancer. ${ }^{2}$ Most studies have focused on the "seed," and it has been suggested that cancer cells secrete various cytokines and proteases, which prompt peritoneal metastasis of cancer cells. ${ }^{3}$ Few studies have been conducted regarding the "soil" of the peritoneum. The physiological state of the peritoneal mesothelium is an effective barrier to block adhesion of tumor cells. ${ }^{4}$ It has been suggested that cancer cells secrete cytokines, which in turn causes pathological changes in peritoneal mesothelial cells. ${ }^{5}$ The changes in the peritoneal microenvironment induce cancer cell metastasis. Peritoneal mesothelial cell fibroblast transformation (MFT) leads to exposure of the stroma below the mesothelial cells, the pathological change provides suitable soil for the dissemination of ovarian cancer cells. ${ }^{6,7}$ The current study investigated the 
morphological changes and mechanism underlying peritoneal mesothelial cells in the process of peritoneal metastasis of ovarian cancer. Our results may provide new molecular targets and a theoretical basis for the prevention and treatment of ovarian cancer peritoneal metastasis.

\section{Materials and methods Chemicals}

Cell culture plastics were obtained from Corning Incorporated (Corning, NY, USA). Recombinant human TGF- $\beta 1$, IL-10, IL-8, TNF- $\alpha$, VEGF, EGF, MMPs, CXCL12, CXCL16, CXCL19, and CXCL21 were obtained from R\&D Systems, Inc. (Minneapolis, MN, USA). Neutralizing antibodies, as well as appropriate isotype-matched control antibodies, were obtained from Abcam (Cambridge, UK). ELISA kits were obtained from R\&D Systems, Inc., and Matrigel was obtained from BD Biosciences (San Jose, CA, USA).

\section{Cell lines and cell cultures}

A human peritoneal mesothelial cell line (HMrSV5) and human ovarian cancer cell lines (SKOV3 and HO8910) were purchased from Shanghai Guandao Biological Engineering Co., Ltd. (Shanghai, China). HMrSV5 cells were cultured in DMEM supplemented with $10 \% \mathrm{FBS}, 100 \mathrm{U} / \mathrm{mL}$ of penicillin, and $100 \mu \mathrm{g} / \mathrm{mL}$ of streptomycin in a $5 \% \mathrm{CO}_{2}$ atmosphere with $70 \%-80 \%$ humidity at $37^{\circ} \mathrm{C}$. SKOV3 and $\mathrm{HO} 8910$ were grown in DMEM supplemented with $10 \% \mathrm{FBS}$ in a $5 \% \mathrm{CO}_{2}$ atmosphere at $37^{\circ} \mathrm{C}$.

All experiments were performed with cell cultures at $60 \%-80 \%$ confluence. To obtain SKOV3 and HO8910 cell-conditioned medium, the cells were seeded and cultivated until $60 \%$ confluence. To obtain HMrSV5 and fibotic HMrSV5 cell-conditioned medium, the cells were seeded and cultivated until $70 \%-80 \%$ confluence. The medium was then replaced with DMEM serum-free medium, and the supernatants were collected after $48 \mathrm{~h}$ of further incubation.

\section{Patients and morphological observations}

The metastatic omental tissues from 10 ovarian cancer patients who were diagnosed by pathological examinations and underwent ovariectomy in Shengjing Hospital of China Medical University from January 2016 to January 2018 were used in this study, of whom five patients were with peritoneal metastasis and five patients were at stage I or II without metastasis and set as controls. The age of five patients with peritoneal metastasis ranged from 27 to 66 years, with a mean age of $45.6 \pm 4.9$ years. The age of five controls ranged from 26 to 64 years, with a mean age of $44.7 \pm 4.9$ years. The samples were sectioned by H\&E staining and compared the section to normal peritoneal tissue samples. This study passed the review of Ethics Committee of Shengjing Hospital of China Medical University before the admission of participants. All participants signed informed consent.

Scanning electron microscopy (SEM) samples were prepared by placing gold nanorod matrices on a flat alumina substrate. The matrix on the substrate was allowed to dry in an open laboratory atmosphere. SEM images were obtained with an environmental field-emission SEM (FEI XL-30 SEM; Philips, Madison, WI, USA) operating with an accelerating voltage of $25 \mathrm{kV}$ and several magnifications at $2000 \times$. Slices of peritoneal tissue were cut at a thickness of $5 \mu \mathrm{m}, \mathrm{H} \& \mathrm{E}-$ stained, and examined under a light microscope.

\section{Western blotting}

The expression of $\alpha$-SMA, E-cad, and cytokeratin- 8 were examined for mesenchymal-epithelial makers. ${ }^{8}$ HMrSV5 cells were cultured to sub-confluent in a $50 \mathrm{~mm}^{2}$ dish with DMEM containing 10\% FBS. The supernatants from the ovarian cancer cell lines (SKOV3 and HO8910) were replaced and serum-free DMEM served as a control. Cells were washed twice with cold PBS and lysed on ice in RIPA buffer with protease inhibitors and quantified using the bicinchoninic acid method. Fifty micrograms of protein lysate from each sample was resolved on $10 \%$ sodium dodecyl sulfate polyacrylamide gels and transferred to polyvinylidene fluoride membranes (EMD Millipore, Billerica, MA, USA) and blocked in 5\% nonfat dry milk in Tris-buffered saline ( $\mathrm{pH}=7.5)$. Membranes were immunoblotted overnight at $4^{\circ} \mathrm{C}$ with antibodies against FM and cytokeratin-8. After overnight incubation, the membranes were incubated with horseradish peroxidase-conjugated secondary antibodies in Tris-buffered saline with Tween 20 (TBST) for $2 \mathrm{~h}$ at room temperature. Finally, the bands were visualized using an enhanced chemiluminescence Western blotting system. All the data of the blot were analyzed by densitometry in the photoshop software and were shown as the mean \pm SD of three experiments.

\section{ELISA}

The HMrSV5 cells were cultured with growth media until reaching $70 \%-80 \%$ confluence and then with serum-free medium to starve the cells overnight. Culture media of fibrotic HMrSV5 were analyzed for CXCL12, CXCL16, CXCL19, and CXCL21. The culture supernatants were collected after incubation for $48 \mathrm{~h}$ and analyzed by ELISA with a human TGF- $\beta 1$, IL-10, IL-8, TNF- $\alpha$, VEGF, EGF, or MMPs' immunoassay in accordance with the ELISA kit instructions. Absorbance was read at $450 \mathrm{~nm}$ using an absorbance micro- 
plate reader. The data on the protein levels were summarized as mean \pm SE of each sample.

\section{Proliferation and migration assays}

SKOV3 and HO8910 cells were plated at a density of $10^{3} /$ well in flat-bottomed 96-well culture plates (Corning Incorporated). After $24 \mathrm{~h}$ of incubation, the medium was replaced with the medium of HMrSV5 (HM-SKOV3/HO8910) supernatants and Fibotic-HMrSV5 (Fibro-HM-SKOV3/HO8910). The preparations were cultured for 5 days, followed by the MTT assay (Sigma-Aldrich Co., St Louis, MO, USA). Absorbance at $579 \mathrm{~nm}$ was taken as a measure of viability. Each experiment was performed in triplicate.

To elucidate the functional role of MFT in ovarian cancer cell migration, we established a MFT model by treating HMrSV5 cells with ovarian cancer cell supernatant for $24 \mathrm{~h}$ with a Transwell chamber assay. The SKOV3 $\left(2 \times 10^{5}\right.$ cells $)$ were seeded into the upper chamber. It was found that MFT transformation of mesothelial cells was induced at the concentration of $100 \mathrm{ng} / \mathrm{mL}$ TGF beta $1 .^{6}$ The cells were induced with medium from the lower chamber to migrate without FBS for $20 \mathrm{~h}$ as follows: 1) HMrSV5 cell suspension in serum-free medium; 2) HMrSV5 cell suspension in SKOV3 medium; and 3) HMrSV5 cell suspension in serum-free medium $+100 \mathrm{ng} / \mathrm{mL}$ of TGF- $\beta 1$. The lower chamber was precoated with $100 \mu \mathrm{L}$ of Matrigel. When HMrSV5 cells reached $80 \%-90 \%$ confluence, SKOV3 cells were seeded into the upper chamber, and after incubation for the indicated times, the cells in upper chamber were gently removed using a cotton-tipped swab. The cells in lower chamber were fixed using methanol and stained with Trypan Blue. The migrated ability was determined by counting the number of penetrating cells in 10 random fields in each well at $\times 200$ magnification.

\section{Statement of ethics}

The protocol was approved by the Research Ethics Committee of China Medical University (Shenyang, China). Written informed consent was provided by all patients.

\section{Results \\ MFT and peritoneal metastasis of ovarian cancer}

To identify the morphological changes in mesothelial cells in the peritoneal metastases of ovarian cancer, we sectioned the peritoneal tissues from five ovarian cancer patients with peritoneal metastasis by H\&E staining and compared the section with normal peritoneal tissue samples. The normal peritoneum surface was one single layer of mesothelial cells, which were cubical and tightly attached to each other; however, the mesothelial cells were spindle and absent with fibroblast-transformed changes in the pathological metastasis peritoneum (Figure 1A and B). The normal and metastatic peritoneal mesothelial cells were also observed under a SEM. The mesothelial cells were arranged tightly and covered with microvilli on the normal peritoneum surface, and the mesothelial cells were undergoing MFT with vanishing superficial microvilli and the formation of local bare areas (Figure $1 \mathrm{C}$ and D).

Compared with the normal cultured mesothelial cell line, HMrSV5, the levels of E-cadherin protein were downregu-
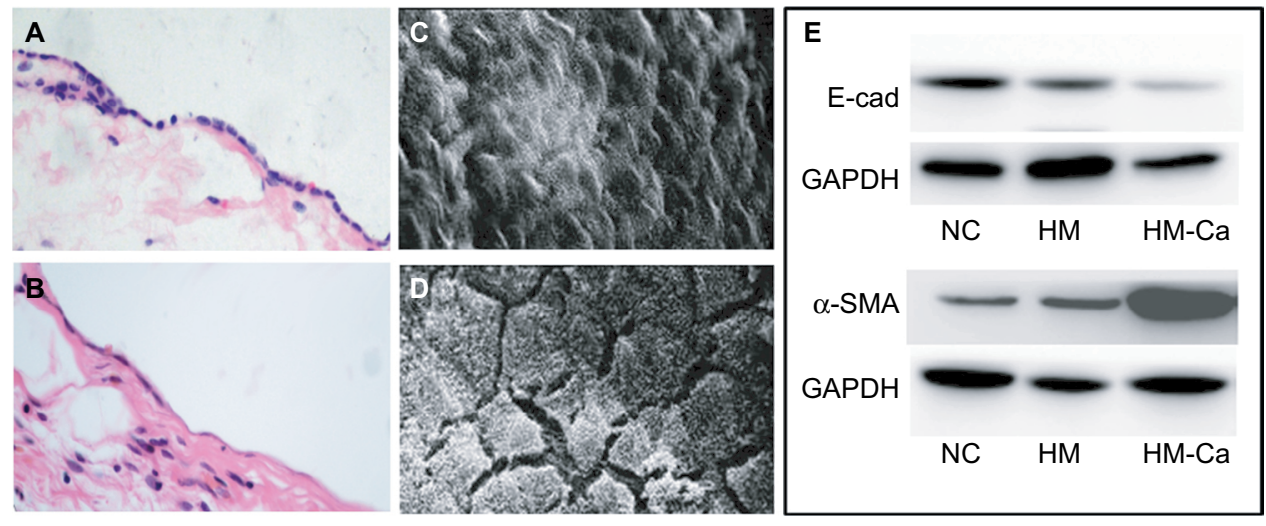

Figure I The morphological and biological changes in mesothelial cells in the peritoneal metastases of ovarian cancer.

Notes: (A) Mesothelial cells of normal omental tissues are closely packed. (B) Mesothelial cells of metastatic omental tissues exhibited fibrosis change with microscopy. Magnification for $(\mathbf{A}$ and $\mathbf{B}) \times 200$. (C) The mesothelial cells of normal omentum were arranged tightly and covered with microvilli on the surface. (D) The MFT mesothelial cells formed local bare areas under SEM. Magnification for (C and D) $\times 2000$. (E) Western blot analysis for the levels of E-cadherin and $\alpha$-SMA proteins in the HMrSV5 and SKOV3 cells. compared with free serum cultured (NC) and normal cultured mesothelial cell line HMrSV5 (HM); the levels of E-cadherin protein were downregulated and the levels of $\alpha$-SMA protein were upregulated in the HMrSV5 cells that were cultured with supernatants from the SKOV3 (HM-Ca).

Abbreviations: MFT, mesothelial cell fibroblast transformation; NC, negative control; SEM, scanning electron microscopy. 
lated and the levels of $\alpha$-SMA protein were upregulated in the HMrSV 5 cells that were cultured with supernatants from the ovarian cancer cell line, SKOV3 (Figure 1E).

\section{TGF- $\beta$ I induces MFT}

We examined the expression of TGF- $\beta 1$, IL-10, IL-8, TNF$\alpha$, VEGF, EGF, and MMPs in ovarian cancer cell lines (SKOV3 and HO8910) using ELISA kits. The levels of TGF- $\beta 1$ and IL-10 were relatively high (415 and $309 \mathrm{pg}$ / $\mathrm{mL} / 10^{6}$ cells, respectively) in SKOV 3 cell lines (Figure $2 \mathrm{~A}$ ), TGF- $\beta 1$ showed the highest level $\left(327 \mathrm{pg} / \mathrm{mL} / 10^{6}\right.$ cells) in the HO8910 cell line (Figure 2B). Then, we examined the cellular morphology of HMrSV5 cells, which were cultured with DMEM pretreated with different concentrations of TGF- $\beta 1(0,10$, and $100 \mathrm{ng} / \mathrm{mL})$. Spindle-like morphology was observed in the presence of TGF- $\beta 1$, which induced MFT (Figure 2C and E). The process involved Western blot analysis, which showed that the expression of $\alpha$-SMA was significantly increased and the expression of E-cadherin and cytokeratin- 8 was significantly decreased in the HMrSV5 cells treated with TGF- $\beta 1(100 \mathrm{ng} / \mathrm{mL})$. These data showed that TGF- $\beta 1$ induced MFT in a time- and dose-dependent manner (Figure 3).

\section{MFT promotes proliferation and migration of ovarian cancer cells}

The HMrSV5 cells were treated with TGF- $\beta 1(100 \mathrm{ng} / \mathrm{mL})$ for $24 \mathrm{~h}$ to induce fibrosis in HMrSV5 cells. The MTT results showed that supernatants of HMrSV5 cell fibrosis (FibroHM-SKOV3/HO8910) stimulated the proliferation of ovarian cancer cells; there was a significant difference in SKOV3 and HO8910 cells compared with serum-free medium $(P<0.01$ for each; Figure 4A and B).

To elucidate the functional role of MFT in ovarian cancer cell invasion, we established a MFT model by treating HMrSV5 cells with ovarian cancer cell supernatant for $24 \mathrm{~h}$ and the SKOV3 cell lines were evaluated in Transwell assays. After $16 \mathrm{~h}$ of incubation for invasion, the wells were harvested and the number of infiltrating cancer cells was counted (Figure 4F). The number of invasive cancer cells was significantly increased in MFT models compared with controls (Figure 4C and E). Mesothelial cell fibrosis promoted the invasiveness of SKOV3 cells. These results showed that the peritoneal fibrosis microenvironment promoted the invasion of ovarian cancer cells and prevented the fibrosis of peritoneal cells that effectively inhibited cancer cell invasion.
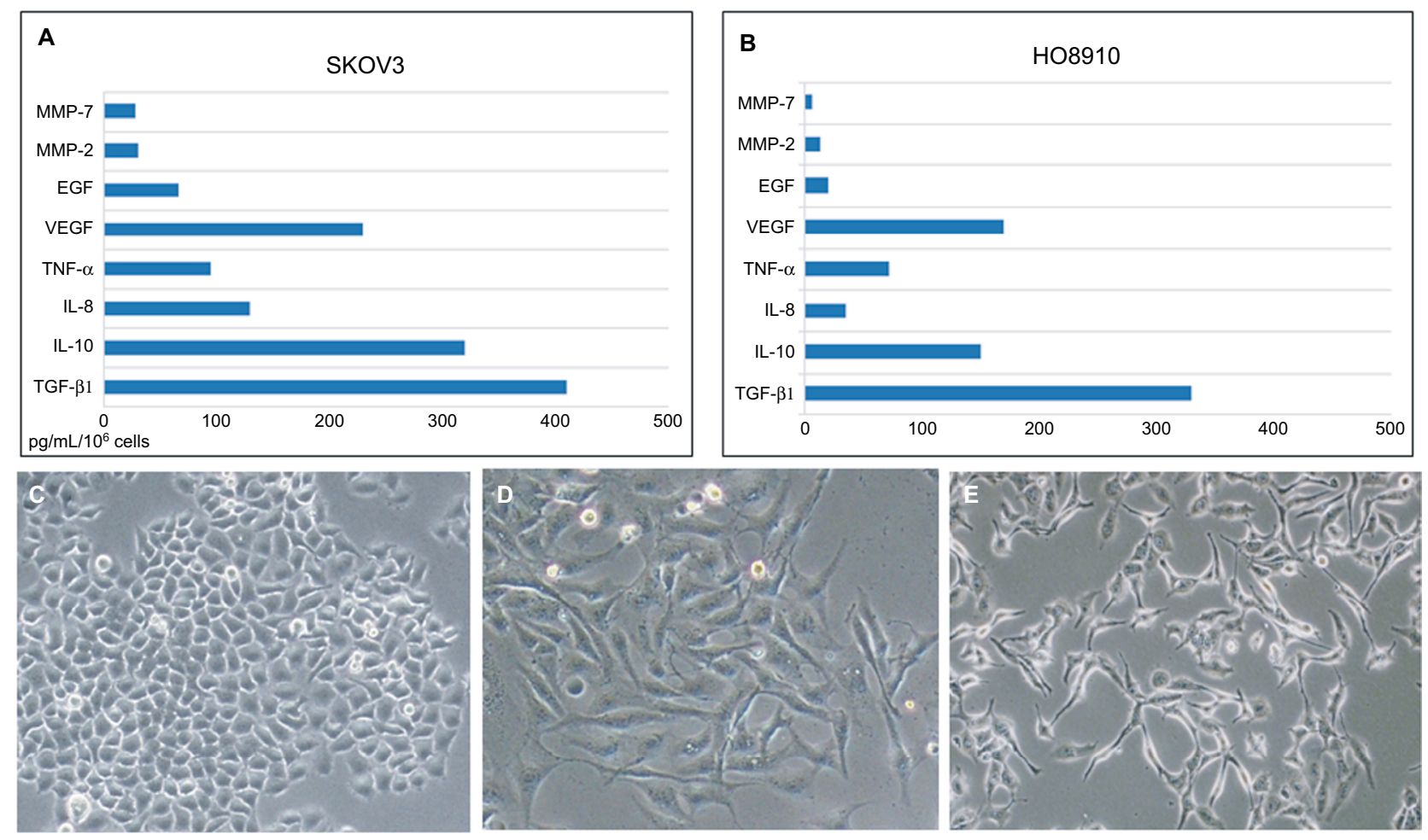

Figure 2 The major cytokines induced mesothelial cell fibroblast transformation.

Notes: The expression of TGF- $\beta$ I, IL-I0, IL-8, TNF- $\alpha$, VEGF, EGF, and MMPs in ovarian cancer cell lines (SKOV3 and HO89I0) using ELISA kits (A and B). The cellular morphology of HMrSV5 cells examined by retreating with different concentrations of TGF- $\beta 1(0,10$, and I00 ng/mL) $(\mathbf{C}-\mathbf{E})$; original magnification $\times 400$. 
A

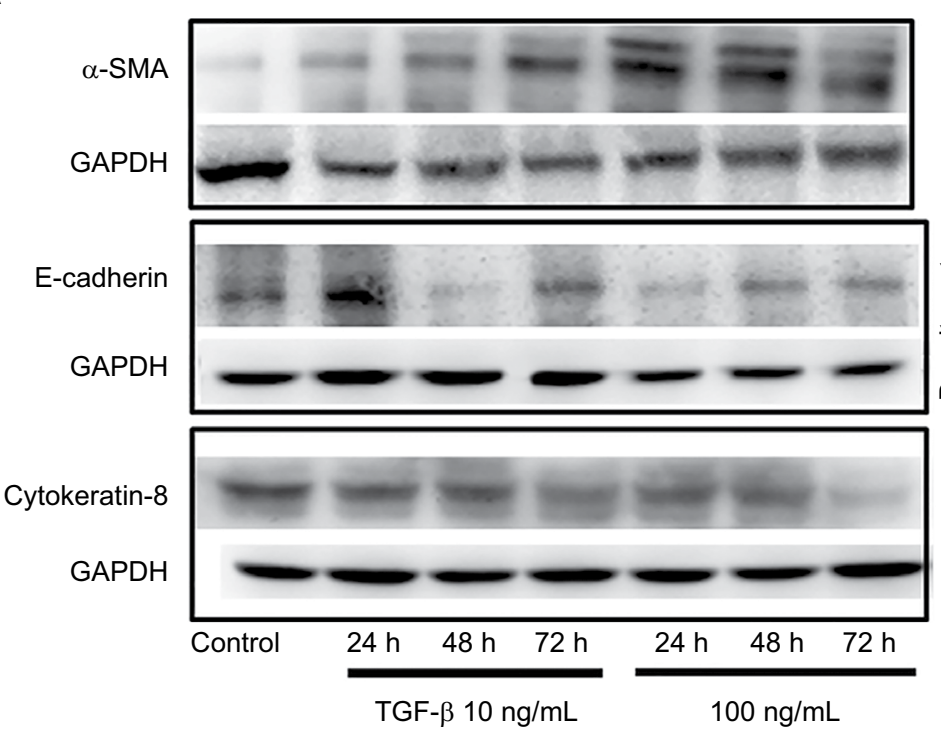

B

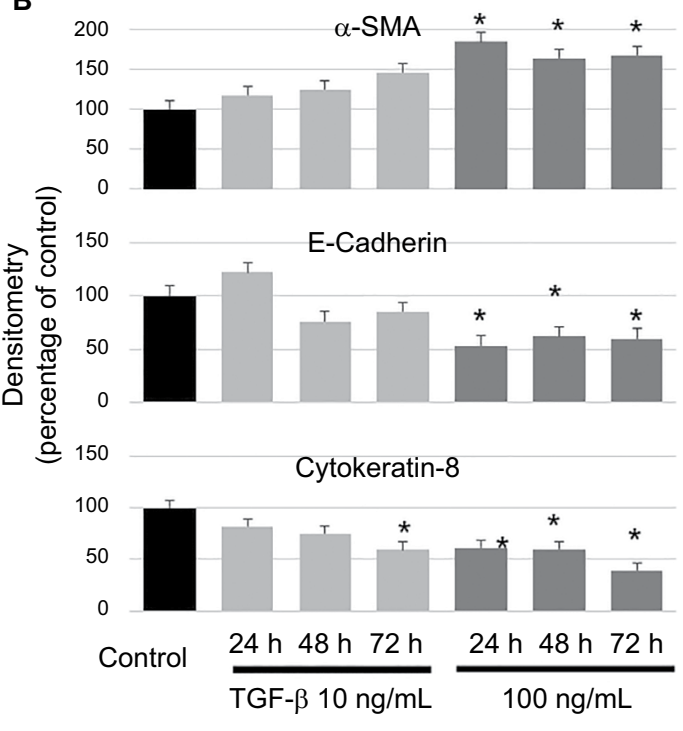

Figure 3 TGF- $\beta$ I could induce MFT in a time- and dose-dependent manner.

Notes: (A) Western blot analysis for the expression of $\alpha$-SMA, E-cadherin, and cytokeratin-8 in the HMrSV5 cells treated with TGF- $\beta$ I (I0 and I00 ng/mL) for $24-72$ h. (B) The Western blot data were quantified with a densitometer, the expression of $\alpha$-SMA was significantly increased, and the expression of E-cadherin and cytokeratin- 8 were significantly decreased in the HMrSV5 cells treated with TGF- $\beta 1(100 \mathrm{ng} / \mathrm{mL})$ compared to the control $(* P<0.05)$.

Abbreviation: MFT, mesothelial cell fibroblast transformation.
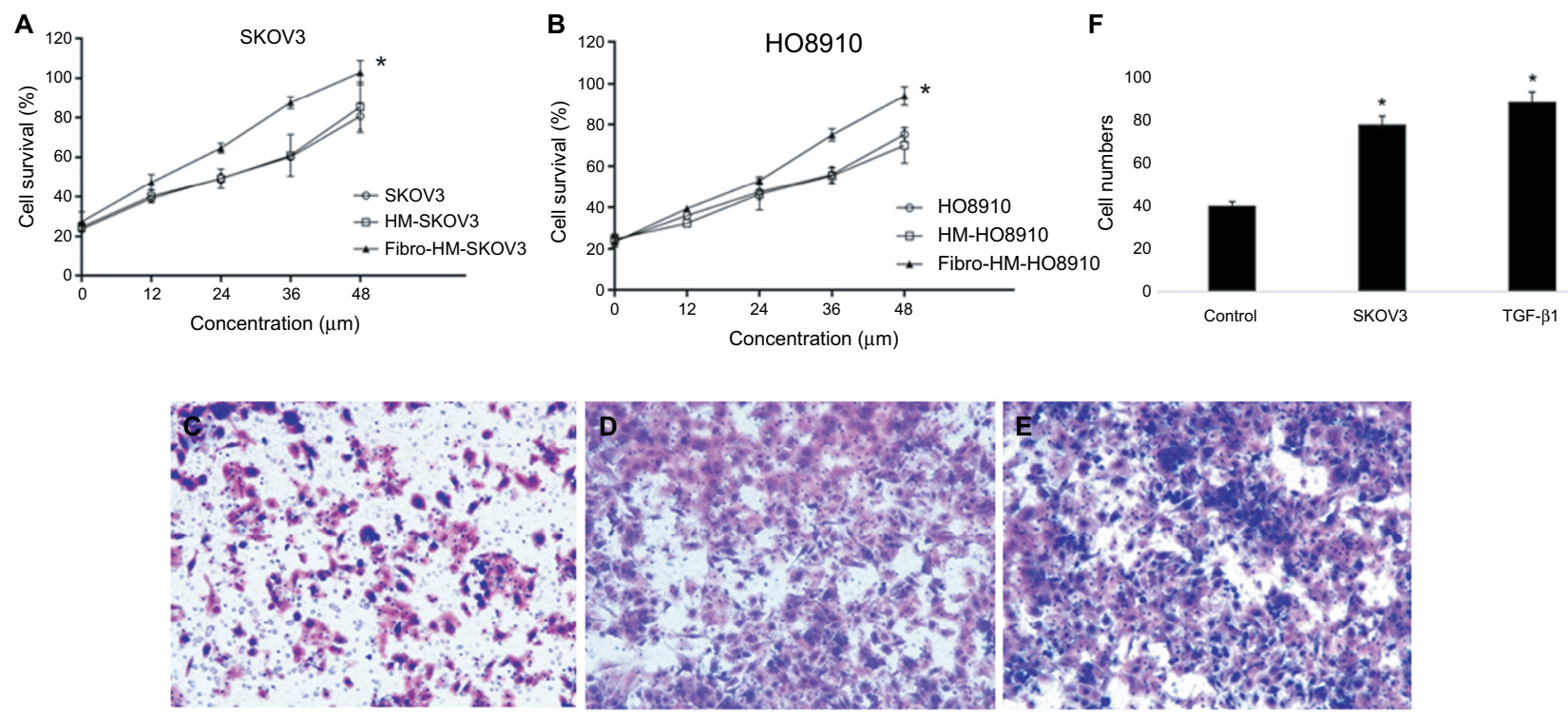

Figure 4 MFT promotes the proliferation and invasion of ovarian cancer cells.

Notes: The MTT results showed that supernatants of fibrotic HMrSV5 cell (Fibro-HM) stimulated the proliferation of ovarian cancer cells. (A) SKOV3 and (B) HO89I0; results are represented as mean \pm SD of three experiments. $* P<0.01$. Cell migration was monitored using a Transwell chamber assay. The SKOV 3 was seeded into the upper chamber. The cells were induced with medium from the lower chamber to migrate without FBS for $20 \mathrm{~h}$ and then replaced with (C) $\mathrm{HMrSV} 5$ cell suspension in serum-free medium, (D) HMrSV5 cell suspension in SKOV3 medium, (E) HMrSV5 cell suspension in serum-free medium $+100 \mathrm{ng} / \mathrm{mL}$ of TGF- $\beta$ I. (F) The number of migrated cancer cells was significantly increased in MFT models (D and E) compared with controls. Magnification $\times 200$.

Abbreviation: MFT, mesothelial cell fibroblast transformation.

\section{Mechanism underlying mesothelial cell promotion of ovarian cancer metastasis}

There was low expression of chemokines in normal HMrSV5 cells and NC group; however, the supernatants of MFT HMrSV5 cells had strong expression of CXCL16 and CXCL12 (142.3 \pm 15.6 and $227.8 \pm 20.3 \mathrm{ng} / \mathrm{mL})$, and SKOV3 cells had high expression of CXCR4, CXCR7, and CXCR6. We examined the levels of ovarian cancer-related chemokines in Fibro-HM and HMrSV5 supernatants. The levels of CXCL16 and CXCL12 were increased in Fibro-HM, 

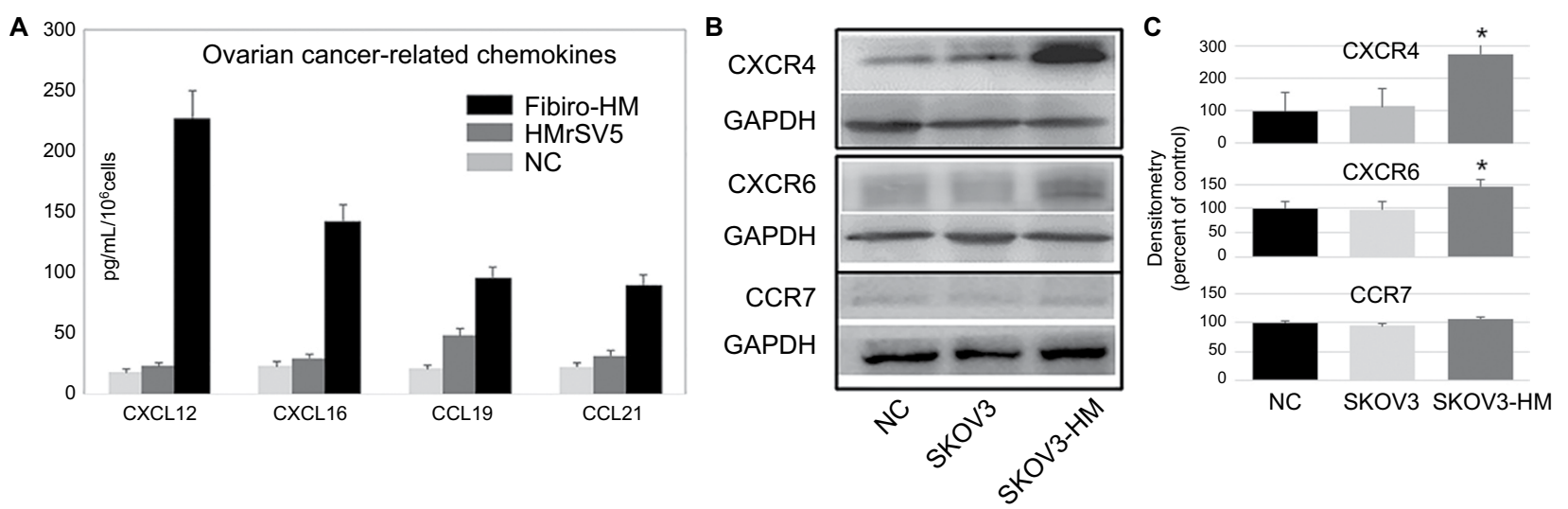

Figure 5 Chemokines of mesothelial cells' promotion of ovarian cancer metastasis.

Notes: (A) The expression of CXCLI2, CXCLI6, CXCL19, and CXCL2I was examined in the supernatants of fibrotic HMrSV5 cell (Fibro-HM) and HMrSV5 by ELISA, the free serum cultured HMrSV5 (NC) was the control group. The data on the protein levels were summarized as mean \pm SE of each sample. (B) The level of chemokine receptor proteins was examined in supernatants of SKOV3 cells. Compared with the free serum cultured (NC) and normal cultured SKOV3 cells (SKOV3), the levels of CXCR4 and CXCR6 were significantly increased in SKOV3 co-cultured with HMrSV5 supernatants (SKOV3-HM). (C) The Western blot data were quantified with a densitometer $(* P<0.05)$.

Abbreviation: NC, negative control.

and the level of CXCL19 was also increased, but without statistical significance (Figure 5A). We then examined the level of chemokine receptor proteins in SKOV3 cells cocultured in HMrSV5 supernatants. The levels of CXCR4 and CXCR6 were significantly increased in SKOV3 co-cultures (Figure 5B).

\section{Discussion}

Ovarian cancer has a definite predilection for metastasis to the peritoneum, but the underlying mechanism is not well understood. ${ }^{8}$ Mesothelial cells are an important constituent of the peritoneal microenvironment. Injury to and changes in peritoneal mesothelial cells morphological structure make the peritoneum a favorable soil for ovarian cancer cells. ${ }^{9,10}$ We have confirmed the MFT process in peritoneal tissues of ovarian cancer patients with peritoneal metastasis and fibrotic mesothelial cell release of chemokines to promote dissemination.

There is an abundance of literature defining the role of TGF- $\beta 1$ in fibrotic disease and wound healing. Recently, several studies have implicated the role of TGF- $\beta 1$ in tumor development and metastasis and some studies have demonstrated that TGF- $\beta 1$ can induce fibrosis of mesothelial cells. ${ }^{11,12}$ The role of TGF- $\beta 1$ in the mechanism underlying MFT inducing implantation has not been investigated. In the current study, we showed high expression of TGF- $\beta 1$ in the supernatant of ovarian cancer cells and established a MFT model. To explore the mechanism underlying MFT inducing metastasis, we analyzed the expression of chemokines and chemokine receptors related to ovarian cancer. CXCL12 and
CXCL16 are members of the CXC family of chemokines. CXCR4 and CXCR6 belong to the CXCR family of chemokine receptors and can only combine with one kind of chemokine. The activation of CXCL12-CXCR4 can cause a series of pathophysiological changes, including angiogenesis, tumor metastasis, and activation of cells. ${ }^{13}$ There are few studies involving the relationship between CXCL12-CXCR4 and breast, liver, and lung cancers. These studies suggested that the CXCL12-CXCR4 axis is involved in the mechanism underlying metastasis. The CXCL16-CXCR6 axis is usually reported in immune system and inflammatory disease, such as hepatitis, atherosclerosis, and AIDS. ${ }^{14}$ Recently, the CXCL16-CXCR6 signaling pathway was implicated in malignant tumors. Some studies have shown that the genesis and development of lung and cervical cancers are closely related to CXCL16-CXCR6. ${ }^{15}$ Some researchers are of the opinion that CXCL16-CXCR6 can promote the proliferation and invasion of gastric cancer cells and is closely related to poor prognosis of gastric cancer patients. ${ }^{16}$

Interestingly, we found that CXCL12 and CXCL16 are the major chemokines in the supernatant of fibrotic mesothelial cells and confirmed that TGF- $\beta 1$ upregulates the CXCL12-CXCR4 and CXCL16-CXCR6 axes of mesothelial cells, which may be a key pathway promoting the infiltration and metastasis of ovarian cancer. Some researchers demonstrated that TGF- $\beta 1$ could activate a TGF- $\beta$ receptor/RAC1/SMAD-dependent signaling pathway in the mesothelial cells that promotes a mesenchymal phenotype and transcriptional upregulation of fibronectin. ${ }^{17,18}$ Our findings indicate that mesothelial cells promote colonization by 
the activation of CXCL12-CXCR4 and CXCL16-CXCR6 axes in fibrotic mesothelial cells during the initial steps of ovarian cancer metastasis and suggest that mesothelial cells actively contribute to metastasis in another key pathway. In recent years, CCL19/CCL21-CXCR7 has been studied in relation to tumors. Studies have revealed that CCL19/ CCL21-CXCR7 take part in the invasion and metastasis of cervical and breast cancers. ${ }^{19,20}$ Our results indicated that CCL19/CCL21-CXCR7 is not involved in the MFT promotion of proliferation and peritoneal metastasis of ovarian cancer.

\section{Conclusion}

Generally, our study delineated the mechanism underlying peritoneal metastasis from the soil in ovarian cancer. These data revealed the important role of MFT and provided an understanding of the mechanism by which MFT promoted the proliferation and peritoneal metastasis of ovarian cancer cells. CXCL12-CXCR4 and CXCL16-CXCR6 may play a critical role in the MFT process. It may lead to develop alternative strategies aimed at preventing and treating metastasis of ovarian cancer.

\section{Acknowledgment}

This study was supported by the National Natural Science Foundation of China (grant no 81772549).

\section{Disclosure}

The authors report no conflicts of interest in this work.

\section{References}

1. Webb PM, Jordan SJ. Epidemiology of epithelial ovarian cancer. Best Pract Res Clin Obstet Gynaecol. 2017;41:3-14.

2. Kalluri R, Weinberg RA. The basics of epithelial-mesenchymal transition. J Clin Invest. 2009;119(6):1420-1428.

3. Cancer Genome Atlas Research N. Integrated genomic analyses of ovarian carcinoma. Nature. 2011;474(7353):609-615.

4. Casey RC, Koch KA, Oegema TR, et al. Establishment of an in vitro assay to measure the invasion of ovarian carcinoma cells through mesothelial cell monolayers. Clin Exp Metastasis. 2003;20(4):343-356.
5. Nakamura M, Ono YJ, Kanemura M, et al. Hepatocyte growth factor secreted by ovarian cancer cells stimulates peritoneal implantation via the mesothelial-mesenchymal transition of the peritoneum. Gynecol Oncol. 2015;139(2):345-354.

6. Lv ZD, Na D, Ma XY, Zhao C, Zhao WJ, Xu HM. Human peritoneal mesothelial cell transformation into myofibroblasts in response to TGFB1 in vitro. Int J Mol Med. 2011;27(2):187-193.

7. Liu XY, Miao ZF, Zhao TT, et al. Milky spot macrophages remodeled by gastric cancer cells promote peritoneal mesothelial cell injury. Biochem Biophys Res Commun. 2013;439(3):378-383.

8. Vergara D, Merlot B, Lucot JP, et al. Epithelial-mesenchymal transition in ovarian cancer. Cancer Lett. 2010;291(1):59-66.

9. Mantha S, Sarasohn D, Ma W, et al. Ovarian vein thrombosis after debulking surgery for ovarian cancer: epidemiology and clinical significance. Am J Obstet Gynecol. 2015;213(2):208:e201-208.

10. Imai $\mathrm{T}$, Horiuchi $\mathrm{A}$, Wang $\mathrm{C}$, et al. Hypoxia attenuates the expression of E-cadherin via up-regulation of SNAIL in ovarian carcinoma cells. Am J Pathol. 2003;163(4):1437-1447.

11. Kariya T, Nishimura H, Mizuno M, et al. TGF- $\beta 1$-VEGF-A pathway induces neoangiogenesis with peritoneal fibrosis in patients undergoing peritoneal dialysis. Am J Physiol Renal Physiol. 2018;314(2): F167-F180.

12. Yuan B, Broadbent JA, Huan J, Yang H. The effects of adipose stem cellconditioned media on fibrogenesis of dermal fibroblasts stimulated by transforming growth factor- $\beta 1$. J Burn Care Res. 2018;39(1):129-140.

13. Guo Q, Gao BL, Zhang XJ, et al. CXCL12-CXCR4 axis promotes proliferation, migration, invasion, and metastasis of ovarian cancer. Oncol Res. 2014;22(5-6):247-258.

14. Peng Y, Ma J, Lin J. Activation of the CXCL16/CXCR6 Axis by TNF- $\alpha$ contributes to ectopic endometrial stromal cells migration and invasion. Reprod Sci. 2018:193371911877679.

15. Huang Y, Zhang J, Cui ZM, Zhao J, Zheng Y. Expression of the CXCL12/ CXCR4 and CXCL16/CXCR6 axes in cervical intraepithelial neoplasia and cervical cancer. Chin J Cancer. 2013;32(5):289-296.

16. Takiguchi G, Nishita M, Kurita K, Kakeji Y, Minami Y. Wnt5a-Ror2 signaling in mesenchymal stem cells promotes proliferation of gastric cancer cells by activating CXCL16-CXCR6 axis. Cancer Sci. 2016;107(3):290-297.

17. Książek K, Bręborowicz A, Jörres A, Witowski J. Oxidative stress contributes to accelerated development of the senescent phenotype in human peritoneal mesothelial cells exposed to high glucose. Free Radic Biol Med. 2007;42(5):636-641.

18. Kenny HA, Chiang CY, White EA, et al. Mesothelial cells promote early ovarian cancer metastasis through fibronectin secretion. $J$ Clin Invest. 2014;124(10):4614-4628.

19. Weitzenfeld P, Kossover O, Körner C, et al. Chemokine axes in breast cancer: factors of the tumor microenvironment reshape the CCR7driven metastatic spread of luminal-A breast tumors. J Leukoc Biol. 2016;99(6):1009-1025.

20. Trimble CL, Clark RA, Thoburn C, et al. Human papillomavirus 16-associated cervical intraepithelial neoplasia in humans excludes CD8 T cells from dysplastic epithelium. J Immunol. 2010;185(11):7107-7114.
Cancer Management and Research

\section{Publish your work in this journal}

Cancer Management and Research is an international, peer-reviewed open access journal focusing on cancer research and the optimal use of preventative and integrated treatment interventions to achieve improved outcomes, enhanced survival and quality of life for the cancer patient. The manuscript management system is completely online and includes

\section{Dovepress}

a very quick and fair peer-review system, which is all easy to use. Visit $\mathrm{http}: / / \mathrm{www}$.dovepress.com/testimonials.php to read real quotes from published authors. 